\title{
Effects of tai chi on catheter management and quality of life in tumor patients with PICC at the intermission of chemotherapy: a non-inferiority randomized controlled trial
}

\author{
Yuehua Ding ${ }^{1 \#}$, Lijun $\mathrm{Ji}^{2 \#}$, Yue $\mathrm{Hu}^{3}$ \\ ${ }^{1}$ Department of PICC Clinic, Hai'an People's Hospital, Hai'an, China; ${ }^{2}$ Department of Breast and Thyroid Surgery, Jiangyin People's Hospital, \\ Jiangyin, China; ${ }^{3}$ Department of Oncology, the Second People's Hospital of Wuxi, Wuxi, China \\ Contributions: (I) Conception and design: Y Ding, Y Hu; (II) Administrative support: L Ji, Y Hu; (III) Provision of study materials or patients: All \\ authors; (IV) Collection and assembly of data: All authors; (V) Data analysis and interpretation: All authors; (VI) Manuscript writing: All authors; (VII) \\ Final approval of manuscript: All authors. \\ \#These authors contributed equally to this work. \\ Correspondence to: Yue Hu. Department of Oncology, Wuxi Second People’s Hospital, Wuxi 214002, China. Email: huyue20193@163.com.
}

Background: Peripherally inserted central catheter (PICC) is widely used in chemotherapy due to its minimal complications and simple wound care. This study explored the effects of tai chi on catheterrelated complications, catheter management ability, and quality of life in tumor patients with PICC at the intermission of chemotherapy.

Methods: This study was an open parallel trial. A total of 98 patients with malignant tumors who underwent long-term chemotherapy with PICC were randomly divided into an observation group (49 cases) and a control group (49 cases). The control group received grip strength exercise for 3 months, while the observation group received 24 simplified tai chi exercises. The coagulation function, thrombosis rate, self-management ability, and quality of life were compared between the two groups before and after the intervention.

Results: After the intervention, the activated partial thromboplastin time (APTT) and prothrombin time (PT) in the observation group were longer than those in the control group, while fibrinogen (FIB) was lower than that in the control group (all $\mathrm{P}<0.05$ ). After the intervention, the total score of self-management ability of the observation group was higher than that of the control group ( $\mathrm{t}=2.038, \mathrm{P}=0.047)$, and the scores of exercises ability and quality of daily life with catheters were significantly increased (all $\mathrm{P}<0.05)$. In terms of quality of life, scores of role-physical (RP), social functioning (SF), mental health $(\mathrm{MH})$ and role-emotional $(\mathrm{RE})$ in the observation group were higher than those in the control group (all $\mathrm{P}<0.05$ ), while there was no statistical difference between the bodily pain (BP) scores of the observation group and the control group $(\mathrm{P}>0.05)$. The incidence of venous thrombosis in the observation group was lower than that in the control group, the difference was statistically significant $\left(\chi^{2}=4.439, \mathrm{P}=0.035\right)$.

Conclusions: Tai chi can prevent PICC thrombosis, reduce PICC-related complications, improve selfmanagement ability, and improve quality of life in at-home patients with long-term PICC.

Keywords: Tai chi; peripherally inserted central catheter (PICC); catheter related complications; catheter management capability; quality of life

Submitted Jun 29, 2020. Accepted for publication Aug 26, 2020.

doi: 10.21037/apm-20-1456

View this article at: http://dx.doi.org/10.21037/apm-20-1456 


\section{Introduction}

Peripherally inserted central catheter (PICC) is a thin and long floating catheter made of biocompatible material (silicone or polyurethane), usually inserted into the forearm, anterior basilar vein, or cephalic vein percutaneously under the guidance of ultrasound. The catheter then enters the central circulation, and the tip of the catheter is usually placed at the superior vena cava or cavoatrial junction (CAJ). Because PICC can reduce the frequency of intravenous injection, is easy to operate, reduces the workload of nurses, and solves the pain of repeated puncture in time to protect peripheral veins and reduce the incidence of tissue necrosis and complications. Therefore, it is often used in critically ill patients and those who require long-term intravenous chemotherapy. Tumor patients $(1,2)$. PICC is often situated in place for $>1$ year, thus long-term placement of PICC could cause venous thrombosis, central line-associated bloodstream infection (CLABSI), and other risks. Johansson et al. (3) pointed out in a systematic review that although PICC has a lower risk of deep vein thrombosis (DVT) when compared with CVC, the risk of catheter blockage is higher. The blood of tumor patients is in a hypercoagulable state. Hisada et al. (4) have shown that the increase in the levels of leukocytes, platelets, tissue factor $(\mathrm{TF}+)$, and microvesicles (MVs) in tumor patients alone, or in combination, is associated with cancer-related venous thrombosis. In addition, tumors present to patients significant psychological, economic, and social burdens. Research has shown (5) that major depressive disorder (MDD) is very common in tumor patients, with the prevalence rate of MDD being 4 times higher than in the general population. Therefore, in order to protect their physical and mental health $(\mathrm{MH})$, it is very important for PICC tumor patients to prevent thrombosis and conduct catheter management during the intermission of chemotherapy.

Tai chi is a traditional Chinese psychophysiological form of exercise which uses soft, slow, and coordinated movements to balance the strength of the limbs. There are different schools of tai chi, but they all share the same key characteristics, namely mindfulness, structural adjustment, and flexibility. Studies $(6,7)$ have shown that tai chi has a positive effect on osteoarthritis, Parkinson's disease, chronic obstructive pulmonary disease (COPD), cognitive function, rehabilitation after stroke and myocardial infarction, and improvement in the quality of life. The tai chi's postural method requires pulling the chest back, keeping the back straight, sinking smoothly and naturally, while relaxing and expanding the rib cage. Therefore it could reduce the pressure on the periphery of the myocardium, increase the systolic pressure difference, output of blood, and raise the oxygen content of the tissues and organs. Maintaining a long-term practice of tai chi can increase the elasticity of blood vessels and theoretically could help prevent thrombosis. Tai Chi has been proven to improve the fatigue of patients with malignant tumors and improve peripheral neuropathy caused by chemotherapy (8). However, its application in patients with malignant tumors undergoing PICC catheterization chemotherapy has not been reported yet. This study is the first to investigate the effect of tai chi on the ability of at home catheter management and the quality of life of patients with PICC catheterization through a randomized controlled trial design method. We present the following article in accordance with the Non-inferiority and Equivalence Trials reporting checklist (available at http://dx.doi.org/10.21037/apm-20-1456).

\section{Methods}

\section{Research object}

A total of 98 patients with malignant tumors who underwent PICC catheterization in the Hai'an People's Hospital from January 2019 to December 2019 were selected as the research object. They were divided into the observation group $(n=49)$ and the control group $(n=49)$ according to a random number table $(1: 1)$. Case inclusion criteria were as follows (I) patients aged 30-75; (II) confirmed by pathological examination as having malignant tumor and requiring long-term chemotherapy; (III) had received PICC catheterization for the first time and were to be discharged with the catheter; (IV) Karnofsky Performance Status (KPS) rating $\geq 60$; (V) No PICC complications when discharged, including phlebitis, catheter obstruction, infection, etc.; (VI) patients or their family members gave informed consent to the study and signed informed consent. Exclusion criteria: (I) previous DVT, thrombophlebitis, and other diseases; (II) existing severe heart, liver, kidney, and other serious diseases; (III) patients who interrupted chemotherapy, died, or chose to go to a non-primary hospital for chemotherapy midway through their treatment; (IV) patients with mental illness or physical, hearing, or language barriers who cannot cooperate. This study was approved by the Ethics Committee of Hai'an People's Hospital (Ethics No. 20190768), and was performed in accordance with the tenets of the Declaration of Helsinki (revised in 2013). All 
subjects agreed to sign the informed consent.

\section{Intervention methods}

This study was performed as an open parallel trial. Before the discharge of each chemotherapy group, the two groups of patients were given guidance by full-time PICC nurses on how to maintain their catheters at home, this involved a demonstration of how the catheters worked, and the provision of PICC catheter maintenance manuals, with information regarding the relevant precautions and the importance of catheter maintenance. Following this induction process, the patients began their chemotherapy intermission home care period. The training for PICC at home self-care was carried out as described below.

Control group: Perform traditional handgrip exercises (9), specifically: (I) each patient was given a grip ball and a detailed training schedule to follow during the chemotherapy intermission; before the patient was discharged, handgrip exercises were taught by professional instructors; (II) the ball was gripped for $10 \mathrm{~s}$, released and rested for $10 \mathrm{~s}$, followed by another $10 \mathrm{~s}$ of gripping the ball. This process was repeated 20 times to constitute 1 set of training, with 1 set performed in the morning, middle of the day, and evening each. A positive mentality and physical strength were maintained for the duration of this training, and it was marked on the schedule after completing training every day. If symptoms of discomfort occurred, they would stop training and immediately contact a member of the full-time nursing staff. The patient could recommence the ball holding training only after having received effective treatment.

Observation group: Tai chi exercise, specifically: simplified tai chi 24 (10) arranged by the National Sports Commission of China (later reorganized as the General Administration of Sport of China) in 1956. Initially, a fulltime coach would lead the practice, and after having learnt, patients could practice at home. The 24 actions are as follows: commencing position, part the wild horse's mane to both sides, white crane spreads its wings, brush knee and twist the hip on both sides, hand strums the lute, repulse the monkey on both sides, grasp the bird's tail left side, grasp the bird's tail right side, single whip, wave hands like clouds, single whip, high pat on horse, kick with the right heel, strike the opponent's temple with fists, turn body and kick with the left heel, squatting and standing on one leg left side, squatting and standing on one leg right side, a fair maiden threads the shuttle both sides, pluck a needle from the sea bottom, open a fan through the back, turn body (wrench, parry, punch), apparent close-up, cross-hands, closing form. The patient performed this sequence of tai chi exercises completely at least 5 times a week during the intermission period of chemotherapy, and each time was expected to be about 60 minutes duration.

\section{Follow-up management}

Patients in the two groups were followed up for 3 months, and those who extubated prematurely due to serious complications had their indwelling time cut off to the time of extubation. We undertook visits every 2 weeks of each participant to observe program fidelity of Tai Chi, and feedback was provided every time. Full-time nursing staff recorded weekly attendance and followed up on unplanned absences. Full-time nursing staff also established PICC home-based nursing records for each patient, including the patient's general information, chemotherapy cycle time, home-based self-care, relevant assessment indicators, and adverse events (AEs). Full-time nursing staff conducted follow-up records by telephone, WeChat, etc., and carried out any relevant evaluations and examinations when patients visited the hospital again.

\section{Evaluation index}

The evaluation index was jointly completed by investigators and researchers who had undergone unified training, and all patients were evaluated before self-care and after 3 months of self-care. The specific indicators of the evaluation index were as below.

\section{General information}

The researchers prepared the general information questionnaires, which included gender, age, marital status, cohabiting family members, level of education, and venipuncture sites.

\section{Self-management ability}

The PICC Self-Management Ability Scale (11) was used for evaluation. The scale has a total of 34 items, including 7 dimensions, namely daily observation of catheters, compliance with catheter maintenance, confidence in catheter management, handling of abnormal events, information access, exercise with catheters, and daily life 
with catheters. Each dimension uses a 5-point Likert scale for scoring, where 1 point means "highly unlikely" and 5 points mean "highly likely". The final score range is 36 to 180 points. The higher the score, the better the selfmanagement ability. The total score is set to 3 levels: the total score $\geq 144$ means good self-management ability, 108 to 144 means medium self-management ability, and $<108$ means poor self-management ability. The internal consistency of content validity of the scale was 0.922 , Cronbach's coefficient alpha was 0.933 , which indicates good reliability and validity.

\section{Quality of life}

The quality of life of patients during PICC catheterization was evaluated using the SF-36 scale (12).

\section{Incidence of venous thrombosis}

Diagnosis was based on the diagnostic criteria for thrombosis (13), meaning there was cyanosis of skin color, elevated temperature, and pain and swelling of the limbs on the affected upper extremities. We used Philips HD 11 color Doppler ultrasound system for the diagnosis of thrombosis, the probe frequency is $5.0-10.0 \mathrm{MHz}$. According to the relevant standards of thrombosis (14), thrombus can be divided into 3 categories: (I) Grade I: there were small hypoechoic clumps in the venous cavity of the catheter, or there were echoic clumps on the outer wall of the catheter. The main manifestation was an isolated echoic clump. Color ultrasound showed that the venous return was smooth, and the stenosis range of the blood vessel cross section was 1-30\%; (II) Grade II: multiple thrombi were present. Color ultrasound showed that the venous blood flow was smooth, and the stenosis range of the blood vessel cross section was $31-50 \%$; (III) Grade III: there was an occlusive thrombus. There were multiple thrombi in the venous cavity and its surroundings, mainly located at the fusion points of the vein, and a large area of the lumen was filled with thrombus.

\section{Blood coagulation index}

Activated partial thromboplastin time (APTT), prothrombin time, and fibrinogen (FIB) levels were detected before and after intervention using an RAC-050 automatic hemagglutination analyzer (Shenzhen Lei Du Life Science Co. Ltd., China).

\section{Statistical analysis}

SPSS 22.0 software (IBM) was used for the statistical analysis of all data. The measurement data was expressed as mean \pm standard deviation, using $t$-test to compare the difference between two independent samples; the count data was expressed by the number of cases and the rate (\%), and the comparison was made by Chi-squared $\left(\chi^{2}\right)$ test; $\mathrm{P}<0.05$ was considered to be statistically significant.

\section{Results}

\section{Comparison of general information of the two groups of patients}

In this study, 98 patients were included, all patients were followed up, and no patients were lost to incomplete followup or having dropped out (Figure 1). The control group included gender: 24 males (48.98\%) and 25 females (51.02\%); age: from $34-70$ years, average age $(50.80 \pm 7.60)$ years; marital status: 43 cases with spouses $(87.76 \%)$, 6 without a spouse (12.24\%); cohabiting family members: 3 cases with children (6.12\%), 42 with spouses $(85.71 \%)$, 4 with parents $(8.16 \%)$; education level: 39 cases $(79.59 \%)$ junior high school and below, 10 cases $(20.41 \%)$ high school and above; venipuncture sites: 28 cases $(57.14 \%)$ were basilic vein, $13(26.53 \%)$ were cephalic vein, and 8 were median vein $(16.33 \%)$. The observation group included gender: 21 males (42.86\%) and 28 females $(57.14 \%)$; age: from $36-72$ years, with an average age of $(53.20 \pm 6.80)$ years; marital status: 45 cases with spouses $(91.84 \%), 4$ without spouses $(8.16 \%)$; cohabiting family members: 5 with children $(10.20 \%), 40$ with spouses $(81.63 \%), 4$ with parents (8.16\%); education level: 37 (75.51\%) junior high school and below, 12 (24.49\%) high school and above; venipuncture sites: 25 cases $(51.02 \%)$ basilic vein, 16 $(32.65 \%)$ cephalic vein, $8(16.33 \%)$ median vein. There was no statistically significant difference in age, gender, marital status, cohabiting family members, education level, and venipuncture sites between the two groups $(\mathrm{P}>0.05)$ (as shown in Table 1), and the two groups were comparable.

\section{Comparison of coagulation function indexes between the two groups}

Before intervention, the APTT, PT, and FIB of the control group were $27.32 \pm 5.01 \mathrm{~s}, 12.44 \pm 1.78 \mathrm{~s}$, and $3.85 \pm 0.73 \mathrm{~g} / \mathrm{L}$, respectively, and the APTT, PT, and FIB of the observation group were $27.34 \pm 5.82 \mathrm{~s}, 12.41 \pm 1.83 \mathrm{~s}$, and $3.88 \pm 0.52 \mathrm{~g} / \mathrm{L}$, 


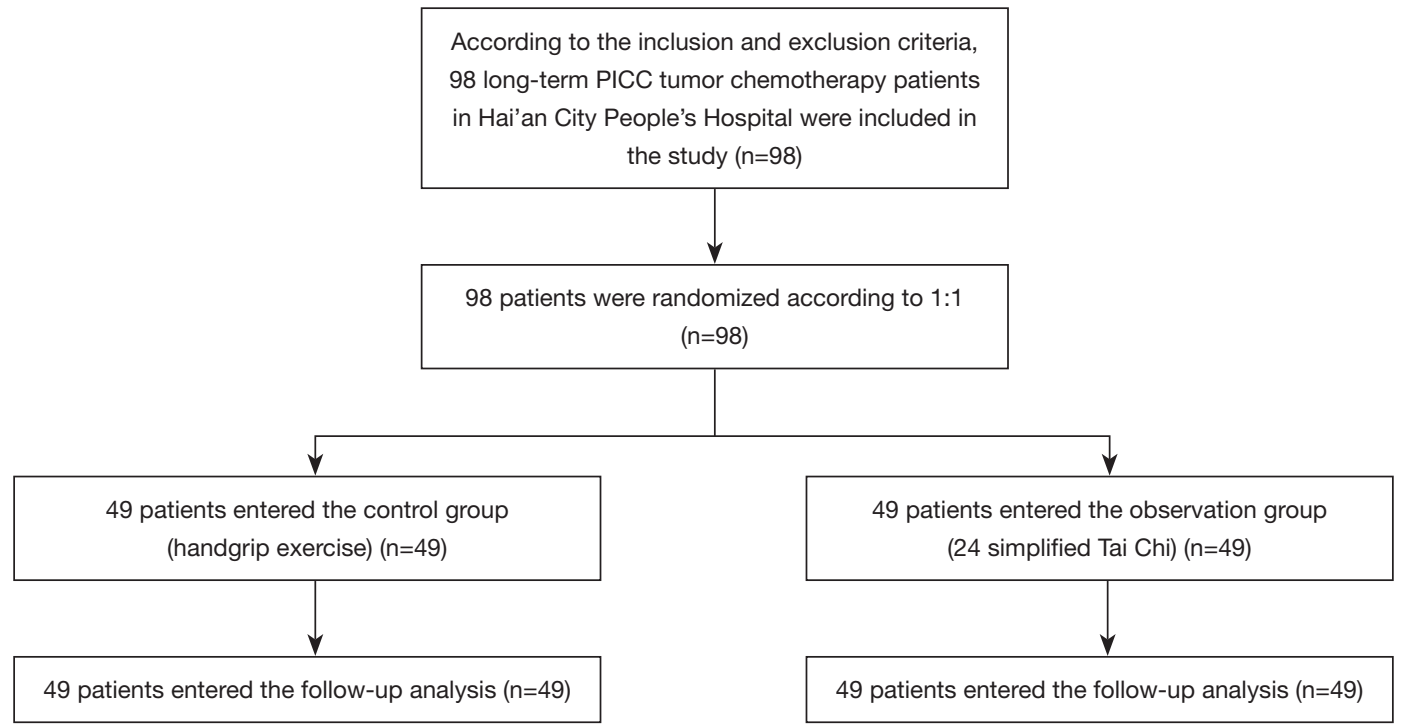

Figure 1 Screening, randomization, follow-up, and analysis of subjects. PICC, peripherally inserted central catheter.

Table 1 Comparison of general information between the two groups (n, \%)

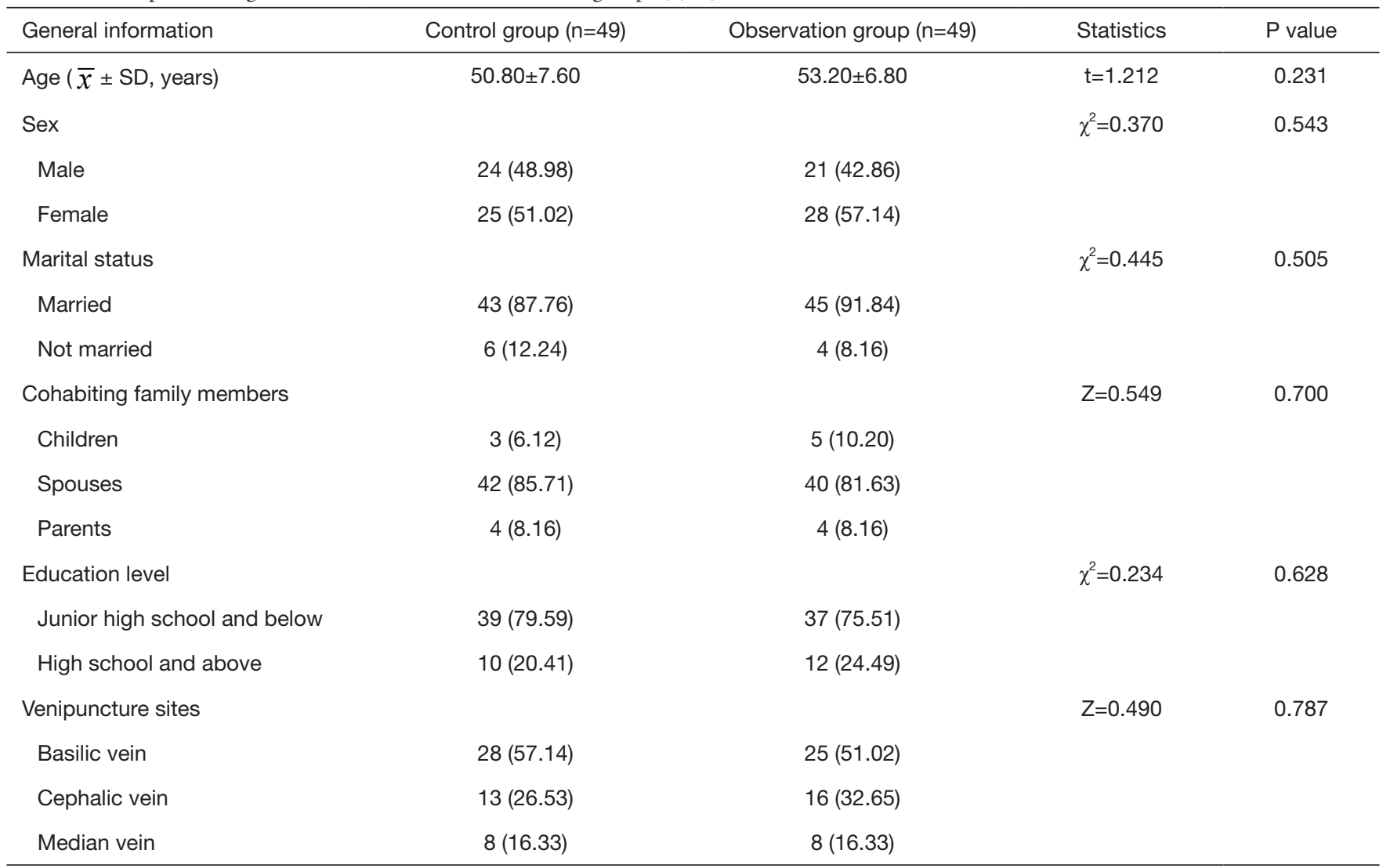


Table 2 Comparison of coagulation function indexes between the two groups ( \pm SD, second)

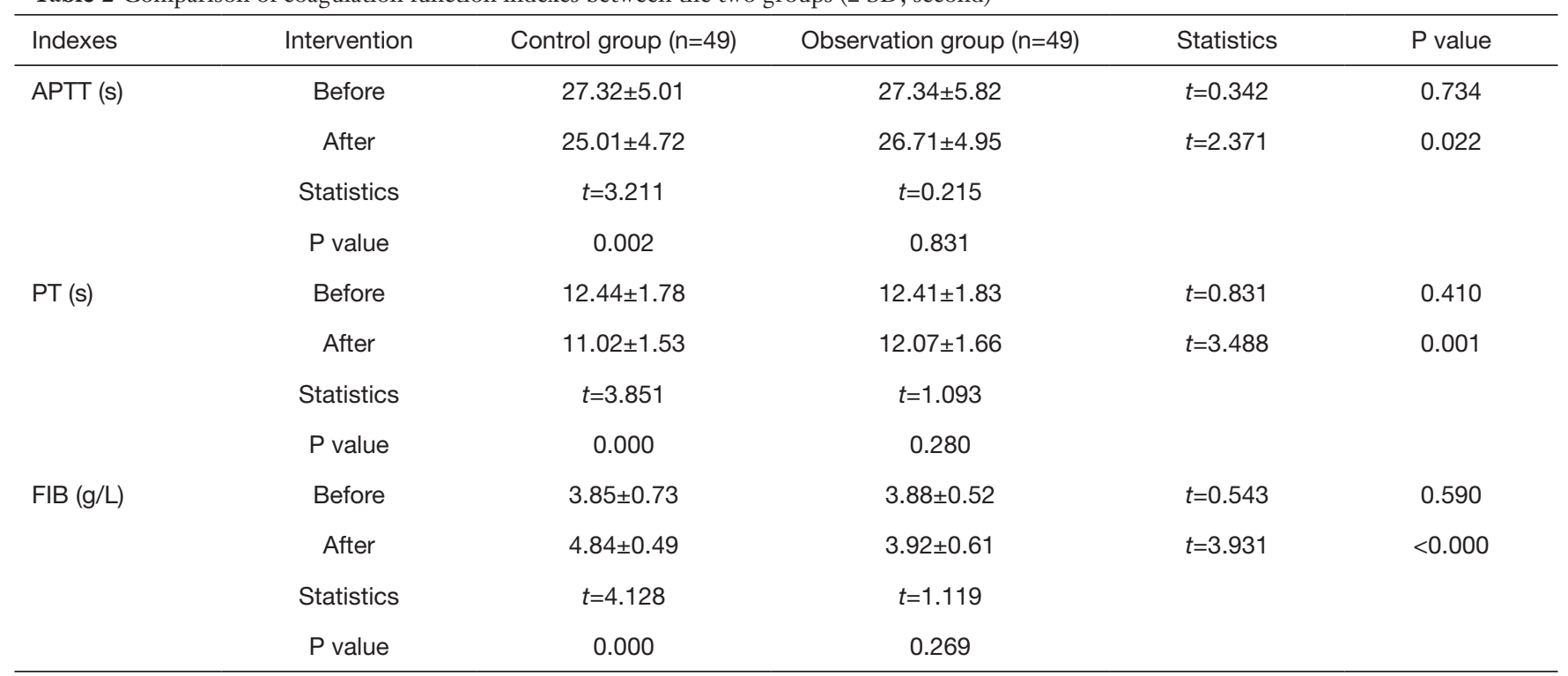

APTT, activated partial thromboplastin time; PT, prothrombin time; FIB, fibrinogen.

respectively. Before intervention, there was no statistical difference in the APTT, PT, and FIB values between the two groups $(\mathrm{P}>0.05)$. After intervention, the APTT, PT, and FIB of the control group were $25.01 \pm 4.72 \mathrm{~s}, 11.02 \pm 1.53 \mathrm{~s}$, and $4.84 \pm 0.49 \mathrm{~g} / \mathrm{L}$, respectively, and the APTT, PT, and FIB of the observation group were $26.71 \pm 4.95 \mathrm{~s}, 12.07 \pm 1.66 \mathrm{~s}$, and $3.92 \pm 0.61 \mathrm{~g} / \mathrm{L}$, respectively. The APTT and PT time of the observation group was longer than that of the control group, and FIB was lower than that of the control group, after intervention, and the differences were statistically significant $(\mathrm{P}<0.05)$. The APTT and PT of the control group were shorter than before the intervention, and the FIB was higher than before the intervention, these differences were statistically significant $(\mathrm{P}<0.05)$; the APTT and $\mathrm{PT}$ of the observation group were shorter than before the intervention, and the FIB was higher than before the intervention, these differences were not statistically significant $(\mathrm{P}>0.05)$, as shown in Table 2.

\section{Comparison of the incidence of venous thrombosis in both groups}

In the control group, there were 6 cases, 4 cases, and 1 case of grade I, II, and III venous thrombosis respectively, and the incidence of venous thrombosis was $22.45 \%$. In the observation group, there were 2 cases, 1 case, and 0 cases of grade I, II, and III venous thrombosis respectively, and the incidence of venous thrombosis was $6.12 \%$. The incidence of venous thrombosis in the observation group was lower than that of the control group, and the difference was statistically significant $\left(\chi^{2}=4.439, P=0.035\right)$, as shown in Table 3.

\section{Comparison of self-management abilities between the two groups}

Before intervention, the total self-management scores of the control group and observation group were $(103.45 \pm 4.18)$ and $(103.72 \pm 4.21)$, respectively, without a statistically significant difference $(\mathrm{P}>0.05)$. The differences of scores for each dimension of self-management between the two groups (daily observation of catheters, compliance with catheter maintenance, confidence in catheter management, handling of abnormal events, access of information, exercise with catheters, and daily life with catheters) were not statistically significant $(\mathrm{P}>0.05)$, as shown in Table 4 .

After intervention, the total self-management scores of the control group and the observation group were $(105.39 \pm 4.66)$ points and $(112.73 \pm 4.89)$ points, respectively. The total score of the observation group was higher than that of the control group, and the difference was statistically significant $(t=2.038, \mathrm{P}=0.047)$. In the observation group, the scores of exercise and daily life with catheters were significantly increased, and the differences were 
Table 3 Comparison of incidence of venous thrombosis between the two groups (n, \%)

\begin{tabular}{lccc}
\hline Grade of venous thrombosis & Control group $(\mathrm{n}=49)$ & Observation group $(\mathrm{n}=49)$ & Statistics \\
\hline I & $6(12.24 \%)$ & $2(4.08 \%)$ & $\chi^{2}=4.439$ \\
II & $4(8.16 \%)$ & $1(2.04 \%)$ & 0.035 \\
III & $1(2.04 \%)$ & $0(0.00 \%)$ & \\
Incidence of venous thrombosis & $22.45 \%$ & $6.12 \%$ & \\
\hline
\end{tabular}

statistically significant compared to the control group $(\mathrm{P}<0.05)$. The control group had no statistically significant difference in the total scores and each dimension score of self-management ability before and after intervention $(\mathrm{P}>0.05)$. The total score of self-management ability of the observation group after intervention and the scores of the ability to exercise and daily life with catheters were significantly higher than before intervention, the differences were statistically significant $(\mathrm{P}<0.05)$, while the self-management ability scores of other dimensions of the observation group before and after intervention were not statistically different $(\mathrm{P}>0.05)$, as shown in Table 4.

\section{Comparison of quality of life between the two groups}

Before intervention, there was no statistically significant difference in the scores of each dimension of quality of life between the two groups $(\mathrm{P}>0.05)$. After intervention, the scores of the four dimensions of role-physical (RP), social functioning (SF), $\mathrm{MH}$, and role-emotional (RE) of the observation group were statistically higher than those of the control group $(\mathrm{P}<0.05)$, but there was no statistical increase in the bodily pain (BP) score between the two groups $(\mathrm{P}>0.05)$. There was no statistically significant difference in the scores of each dimension of quality of life before and after intervention in the control group $(\mathrm{P}>0.05)$. The scores of the four dimensions of SF, RP, RE, and $\mathrm{MH}$ of the observation group after intervention were significantly higher than before, and the differences were statistically significant $(\mathrm{P}<0.05)$, but there was no statistical increase in the $\mathrm{BP}$ score before and after intervention $(\mathrm{P}>0.05)$, as shown in Table 5.

\section{Discussion}

Since PICC was first reported for long-term total parenteral nutrition in 1975, the safety of this technique has been confirmed. Compared with traditional CVC,
PICC is easy to insert, can be retained for an extended time, which can reduce blood flow infections caused by frequent replacement, and is low cost. At the same time, we acknowledge that any indwelling catheter poses the risk of venous thromboembolism and CLABSI. The PICC inserted into the vein is a foreign body, which may cause vascular endothelial damage, additionally, tumor patients have high blood viscosity. Thromboembolism is a common complication for PICC patients, and in severe cases is potentially life-threatening $(15,16)$. Cancer patients can often require long-term intravenous drug chemotherapy. Studies have shown that (17) PICC is recommended for chemotherapy in cancer patients in the medium and long term due to the related AEs rates being lower with PICC than with traditional CVC. Therefore, PICC is the most commonly used and cost-effective venous access in the clinical setting. When improper care (failure to flush the tube and seal the tube in a timely manner) and the hypercoagulable state of the tumor patients occur simultaneously, the PICC catheter easily can become blocked. During the hospital stay, nursing staff manage the PICC patients through the bundle nursing strategy (18), which analyzes and takes measures to avoid the risks that may cause the patients' PICCs to become blocked. Thus self-management of PICC for patients at the intermission of chemotherapy is particularly important (19). PICC daily management (20) includes recognizing any problems, handling of exceptions, patient confidence, inspection of the tube and membrane application, and observation of the movements of the PICC in the indwelling arm, but it does not include maintenance of the catheter. These daily management practices could reduce the occurrence of AEs. This study was the first to explore the effect of tai chi exercise on the ability to self-manage and quality of life of PICC patients.

In this study, a randomized controlled experiment (RCT) design was used to divide tumor patients into the control group (handgrip exercise) and the observation group 
Table 4 Comparison of self-management abilities between the two groups ( $\bar{x} \pm \mathrm{SD}$, points)

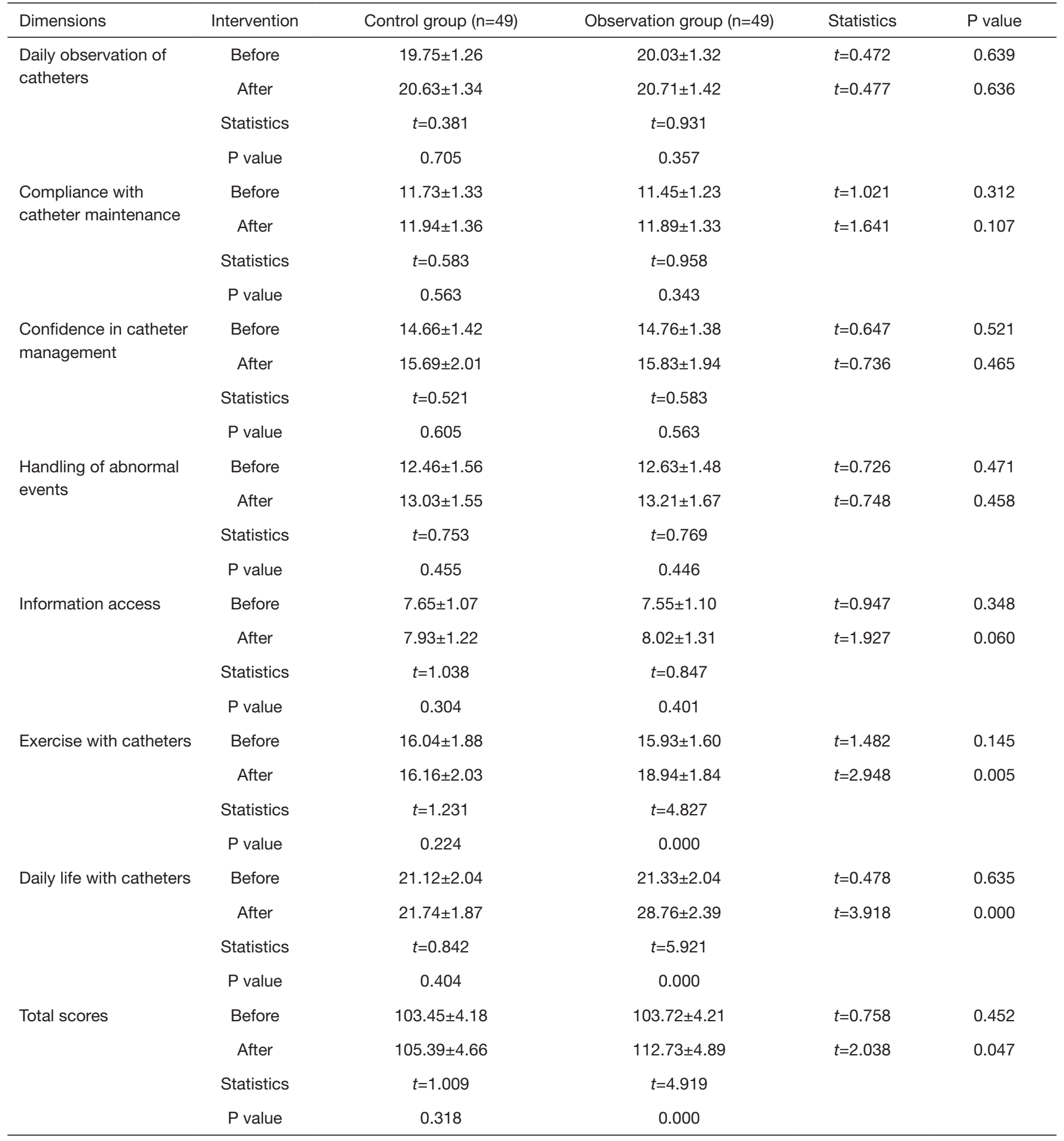


Table 5 Comparison of quality of life between the two groups of patients ( $\bar{x} \pm \mathrm{SD}$, points)

\begin{tabular}{|c|c|c|c|c|c|}
\hline Dimensions & Intervention & Control group $(n=49)$ & Observation group $(n=49)$ & Statistics & $P$ value \\
\hline \multirow{3}{*}{ SF } & After & $53.74 \pm 15.2$ & $72.5 \pm 12.5$ & $t=2.482$ & 0.017 \\
\hline & Statistics & $t=0.264$ & $t=2.101$ & & \\
\hline & $P$ value & 0.793 & 0.041 & & \\
\hline \multirow{3}{*}{$\mathrm{RP}$} & After & $34.52 \pm 10.41$ & $54.73 \pm 15.83$ & $t=3.012$ & 0.004 \\
\hline & Statistics & $t=0.836$ & $t=2.948$ & & \\
\hline & $P$ value & 0.407 & 0.005 & & \\
\hline \multirow[t]{2}{*}{ BP } & Before & $62.83 \pm 14.73$ & $67.88 \pm 12.52$ & $t=0.738$ & 0.464 \\
\hline & $P$ value & 0.852 & 0.052 & & \\
\hline \multirow[t]{4}{*}{ RE } & Before & $50.72 \pm 8.62$ & $48.62 \pm 9.01$ & $t=1.002$ & 0.321 \\
\hline & After & $49.15 \pm 7.66$ & $63.81 \pm 10.91$ & $t=2.031$ & 0.048 \\
\hline & Statistics & $t=1.039$ & $t=2.381$ & & \\
\hline & $P$ value & 0.304 & 0.021 & & \\
\hline \multirow[t]{3}{*}{$\mathrm{MH}$} & Before & $65.82 \pm 9.76$ & $68.09 \pm 10.77$ & $t=0.834$ & 0.408 \\
\hline & After & $68.41 \pm 10.73$ & $78.77 \pm 10.29$ & $t=2.831$ & 0.007 \\
\hline & Statistics & $t=1.123$ & $t=2.829$ & & \\
\hline
\end{tabular}

$\mathrm{SF}$, social functioning; RP, role-physical; BP, bodily pain; RE, role-emotional; $\mathrm{MH}$, mental health.

(24 simplified tai chi) at random. The results suggest that tai chi exercise can prolong the APTT and PT and decrease the level of FIB in PICC patients; it can also significantly reduce the incidence of venous thrombosis in patients with PICC $(\mathrm{P}<0.05)$. Tai Chi is a soft and slow aerobic exercise, which includes the practices of following the movement of the hands with the eyes, one-leg support, gravity conversion, limb coordination, and abdominal breathing. Compared with ordinary handgrip exercises, tai chi pays more attention to whole body coordination. Research has shown (21) that regularly performing tai chi can enhance the flexibility of facet joints, prevent falls, and improve fatigue and depression. Moreover, it can assist in the treatment of chronic diseases, including breast cancer, rheumatoid arthritis, cardiovascular disease, high blood pressure, and osteoporosis, amongst others. The 24 simplified tai chi is easy to encourage participation in and is widely used in the adjuvant treatment of the above chronic diseases. The mechanism by which tai chi could be useful in preventing thrombosis may be related to the following: Firstly, the stretching exercises involved may increase blood supply to the organs and improve arterial compliance. Secondly, it could increase the body's oxygen supply through aerobic exercise, thus reduce blood viscosity and relieve the hypercoagulation state of the serum. Thirdly through relaxing and contracting muscles, it could accelerate the rate of blood flow, enhancing the elasticity of the blood vessel wall (22).

Our study also found that the self-management ability of the observation group was significantly better than that of the control group, especially in the exercise and daily life with catheters, the differences were statistically significant $(\mathrm{P}<0.05)$. Tai chi is a traditional Chinese method of exercising for health. Compared with handgrip exercise, 
patients who perform tai chi are more likely to improve fatigue, so they pay closer attention to how to exercise with catheters and their daily life, thus improve their selfmanagement.

This study also found that after intervention, the scores of SF, RP, RE, and $\mathrm{MH}$ in the observation group were significantly higher than those in the control group $(\mathrm{P}<0.05)$. This shows that tai chi exercise can contribute positively to mental and physical health along with the social element of practice for patients with PICC. This is similar to the findings of a meta-analysis (23) that investigated the impact of tai chi on the quality of life of tumor patients. The study revealed that tai chi could improve quality of life physically and psychologically. There is moderate evidence that tai chi can lower cortisol and corticotropin-releasing factor (CRF) levels and improve limb function. This may be related to the following factors: first, the 24 simplified tai chi is easy to learn and can balance the strength of the limbs; second, tai chi has a positive impact on tumor patients' perception of happiness, which is due to the sharing of mutual interests and hobbies between the members of the tai chi participant group; third, tai chi requires a combination of posture and balance, which positively affects the functioning of the musculoskeletal and central nervous systems. Tai chi emphasises slow and deep breathing exercises, which can increase movement of the diaphragm, chest, and abdomen, potentially improving blood circulation. With all the above elements considered, tai chi has a positive effect on improving the patient's quality of life.

This study has the following limitations: (I) a potential limitation of this study is that the sample size of patients is a bit small, but each test indicates that there is a difference between the two groups. We will carry out a larger sample study in future studies, focusing on exploring the effects of tai chi on catheter management and quality of life in tumor patients with PICC at the intermission of chemotherapy. (II) Due to the characteristics of the two sets of interventions, this study was designed as an open study, it could not be designed blindly. In theory, this may have increased the risk of bias in subjective outcomes such as self-management ability and quality of life scores, but it had little effect on the assessment of PICC complications.

\section{Conclusions}

In conclusion, tai chi exercise could prevent PICC thrombosis, reduce PICC-related complications, improve self-management ability, and improve the quality of life for at-home patients with long-term PICC catheterization. Tai chi (24 simplified) is easy to learn and it is recommended to assist with self-catheter management at home for tumor patients with long-term PICC catheterization.

\section{Acknowledgments}

Funding: None.

\section{Footnote}

Reporting Checklist: The authors have completed the Noninferiority and Equivalence Trials reporting checklist. Available at http://dx.doi.org/10.21037/apm-20-1456

Data Sharing Statement: Available at http://dx.doi. org/10.21037/apm-20-1456

Conflicts of Interest: All authors have completed the ICMJE uniform disclosure form (available at http://dx.doi. org/10.21037/apm-20-1456). The authors have no conflicts of interest to declare.

Ethical Statement: The authors are accountable for all aspects of the work in ensuring that questions related to the accuracy or integrity of any part of the work are appropriately investigated and resolved. This study was performed in compliance with the tenets of the Declaration of Helsinki (as revised in 2013) and was approved by the Ethics Committee of Hai'an People's Hospital (Ethics No. 20190768). All subjects agreed and signed informed consent.

Open Access Statement: This is an Open Access article distributed in accordance with the Creative Commons Attribution-NonCommercial-NoDerivs 4.0 International License (CC BY-NC-ND 4.0), which permits the noncommercial replication and distribution of the article with the strict proviso that no changes or edits are made and the original work is properly cited (including links to both the formal publication through the relevant DOI and the license). See: https://creativecommons.org/licenses/by-nc-nd/4.0/.

\section{References}

1. Robinson A, Souied $\mathrm{O}$, Bota $\mathrm{AB}$, et al. Optimal vascular access strategies for patients receiving chemotherapy for early-stage breast cancer: a systematic review. Breast 
Cancer Res Treat 2018;171:607-20.

2. Zochios V, Umar I, Simpson N, et al. Peripherally inserted central catheter (PICC)-related thrombosis in critically ill patients. J Vasc Access 2014;15:329-37.

3. Johansson E, Hammarskjöld F, Lundberg D, et al. Advantages and disadvantages of peripherally inserted central venous catheters (PICC) compared to other central venous lines: a systematic review of the literature. Acta Oncol 2013;52:886-92

4. Hisada Y, Mackman N. Cancer-associated pathways and biomarkers of venous thrombosis. Blood 2017;130:1499-506.

5. Bortolato B, Hyphantis TN, Valpione S, et al. Depression in cancer: The many biobehavioral pathways driving tumor progression. Cancer Treat Rev 2017;52:58-70.

6. Taylor-Piliae RE, Finley BA. Tai Chi exercise for psychological well-being among adults with cardiovascular disease: A systematic review and meta-analysis. Eur J Cardiovasc Nurs 2020;1474515120926068.

7. Huston P, McFarlane B. Health benefits of tai chi: What is the evidence? Can Fam Physician 2016;62:881-90.

8. Brami C, Bao T, Deng G. Natural products and complementary therapies for chemotherapy-induced peripheral neuropathy: A systematic review. Crit Rev Oncol Hematol 2016;98:325-34.

9. Pagonas N, Vlatsas S, Bauer F, et al. Aerobic versus isometric handgrip exercise in hypertension: a randomized controlled trial. J Hypertens 2017;35:2199-206.

10. Wu F, Song EF, Bao Y, et al. Tai chi for the treatment of type 2 diabetes. Chin J Phys Med Rehabil 2010;32:205-7.

11. Ma D, Cheng K, Ding P, et al. Self-management of peripherally inserted central catheters after patient discharge via the WeChat smartphone application: A systematic review and meta-analysis. PLoS One 2018; 13:e0202326.

12. Treanor C, Donnelly M. A methodological review of the Short Form Health Survey 36 (SF-36) and its derivatives among breast cancer survivors. Qual Life Res 2015;24:339-62.

13. Wada H, Gabazza EC, Asakura H, et al. Comparison of diagnostic criteria for disseminated intravascular coagulation (DIC): diagnostic criteria of the International Society of Thrombosis and Hemostasis and of the Japanese Ministry of Health and Welfare for overt DIC. Am J Hematol 2003;74:17-22.

14. Watson HG, Keeling DM, Laffan M, et al. Guideline on aspects of cancer-related venous thrombosis. $\mathrm{Br} \mathrm{J}$ Haematol 2015;170:640-8.

15. Venkataraman ST. To PICC or Not to PICC, That Is the Question! Pediatr Crit Care Med 2018;19:1168-9.

16. Patel GS, Jain K, Kumar R, et al. Comparison of peripherally inserted central venous catheters (PICC) versus subcutaneously implanted port-chamber catheters by complication and cost for patients receiving chemotherapy for non-haematological malignancies. Support Care Cancer 2014;22:121-8.

17. Campagna S, Gonella S, Berchialla P, et al. Can Peripherally Inserted Central Catheters Be Safely Placed in Patients with Cancer Receiving Chemotherapy? A Retrospective Study of Almost 400,000 Catheter-Days. Oncologist 2019;24:e953-9.

18. Li CC, Hu J, Su XN, et al. Research progress of bundles nursing and it's application in surgical nursing. Chin J Mod Nurs 2017;23:3813-6.

19. Yang PH, Hsu HC, Chiang CC, et al. Improving the Care Accuracy of Percutaneously Inserted Central Catheters Using Objective Structured Clinical Examination. Hu Li Za Zhi 2016;63:112-8.

20. Chen J, Zhao H, Xia Z, et al. Knowledge, attitude, and practice toward the daily management of PICC in critically ill cancer patients discharged from intensive care units. Cancer Manag Res 2018;10:1565-74.

21. Taylor E, Taylor-Piliae RE. The effects of Tai Chi on physical and psychosocial function among persons with multiple sclerosis: A systematic review. Complement Ther Med 2017;31:100-8.

22. Lu X, Hui-Chan CW, Tsang WW. Tai Chi, arterial compliance, and muscle strength in older adults. Eur J Prev Cardiol 2013;20:613-9.

23. Ni X, Chan RJ, Yates P, et al. The effects of Tai Chi on quality of life of cancer survivors: a systematic review and meta-analysis. Support Care Cancer 2019;27:3701-6.

(English Language Editor: J. Jones)

Cite this article as: Ding Y, Ji L, Hu Y. Effects of tai chi on catheter management and quality of life in tumor patients with PICC at the intermission of chemotherapy: a non-inferiority randomized controlled trial. Ann Palliat Med 2020;9(5):3293-3303. doi: 10.21037/apm-20-1456 